\title{
Comparison of Bone Mineral Density and Markers of Bone Turnover in Osteoporotic Women after 6-Month Treatment with Alendronate or Bazedoxifene: A Randomized Controlled Trial
}

\author{
Hee Soo Han' ${ }^{1}$ Sung Hee Cho' ${ }^{2}$, Moon Seok Park', Ki Hyuk Sung ${ }^{1}$, Kyoung Min Lee' \\ 'Department of Orthopedic Surgery, Seoul National University Bundang Hospital, Seoul National University College of Medicine, \\ Seongnam; \\ ${ }^{2}$ Department of Orthopedic Surgery, Gyeongsang National University Hospital, Gyeongsang National University School of Medicine, Jinju, \\ Korea
}

Corresponding author

Kyoung Min Lee

Department of Orthopedic Surgery, Seoul

Nationl University Bundang Hospital,

82 Gumi-ro 173 beon-gil, Bundang-gu,

Seongnam 13620, Korea

Tel: +82-31-787-7205

Fax: +82-31-787-4056

E-mail: 0asis100@empal.com

Received: January 12, 2021

Revised: March 13, 2021

Accepted: March 18, 2021
Background: In a randomized controlled trial, we compared the bone mineral densities (BMDs) and blood markers of bone turnover during short-term treatment of osteoporotic women with bisphosphonate alendronate or bazedoxifene, a selective estrogen receptor modulator. Methods: Ten and eleven patients were randomized to the alendronate and bazedoxifene groups, respectively. BMDs were measured before and after 6 months of treatment. Blood tests were used to measure the levels of osteocalcin (OC), Cterminal telopeptide of type I collagen (CTX), vitamin D3, and parathyroid hormone pretreatment and after 3 and 6 months of treatment. The variables were compared statistically. Results: The alendronate group showed decreases in blood levels of both $\mathrm{OC}$ and CTX during the study period ( $P<0.001$ and $P=0.002$, respectively), while the bazedoxifene group had a decrease only in OC levels $(P=0.012)$. After 6 months of treatment, BMDs significantly increased in the alendronate group at multiple bone sites, including the L1-4 lumbar vertebrae, femur trochanter, and total femur. However, there was no significant increase in BMD in the bazedoxifene group. BMDs were not significantly different between the 2 groups. Conclusions: Patients treated with alendronate showed more rapid suppression of markers of bone turnover and higher BMD than those treated with bazedoxifene during a short-term regime. Considering the effects and complications of each medication, the relationship between bone turnover rate and bone quality will need to be investigated in future studies.

Key Words: Alendronate · Bone density · Bone resorption · Osteogenesis · Selective estrogen receptor modulators

\section{INTRODUCTION}

Mineral Research

This is an Open Access article distributed under the terms of the Creative Commons Attribution Non-Commercial License (https://creativecommons.org/licenses/by-nc/4.0/) which permits unrestricted non-commercial use, distribution, and reproduction in any medium, provided the original work is properly cited.

The structure and function of the bone are achieved by a constant dynamic process and equilibrium between bone formation and resorption, and the quality and quantity of bone tissues are balanced by this equilibrium.[1] Osteoporosis is a systemic disease characterized by decreased bone mass and microstructural deterioration of bone tissue that increases fragility and susceptibility to fracture.[2] Al- 
though the measurement of bone mineral density (BMD) performed using dual energy X-ray absorptiometry (DXA) is the current gold standard for the diagnosis of osteoporosis, it is only useful for detecting structural changes that are greater than $30 \%$ of normal.[3]

Bone metabolism can be measured by biochemical markers that can detect structural changes earlier than a DXA scan. Markers for bone resorption reflect the activity of osteoclasts and include the $\mathrm{N}$-terminal telopeptide and Cterminal telopeptide (CTX) of type 1 collagen. Osteocalcin (OC), alkaline phosphatase, C-terminal propeptide, and Nterminal propeptide are markers of bone formation which reflect osteoblastic activity. Furthermore, blood levels of parathyroid hormone (PTH) and 25-dihydroxy-vitamin D3 are useful for evaluating the status of bone turnover.[4]

Therapeutics that modify bone turnover equilibrium are used to treat osteoporosis. Anti-resorptive agents are effective and widely used. Out of many medication options, anti-resorptive agents are commonly prescribed to postmenopausal women. Two of the most common anti-resorptive agents are oral bisphosphonates such as alendronate, risedronate, and ibandronate, and selective estrogen receptor modulators (SERMs).[5] Anti-resorptive agents decrease the number, activity, and life span of osteoclasts. However, it should be noted that not all anti-resorptive drugs act similarly that can lead to different treatment results and complications.[6] This is the first study that directly compared the effects of alendronate and bazedoxifene on bone turnover markers.

The objective of this study was to compare the efficacy of alendronate and bazedoxifene in osteoporotic women during 6 months of treatment using DXA scans to detect changes in BMD and measurements of blood markers for bone turnover.

\section{METHODS}

\section{Study participants}

This prospective study from October 2014 to April 2016 was approved by the Institutional Review Board at our hospital. Informed consent was obtained from each participant after a detailed explanation of the study. The inclusion criteria were (1) female gender; (2) T-score $\leq-2.5$ using DXA; (3) age from 50 to 80 years; and (4) naive to osteoporosis medication. Exclusion criteria were (1) women with endometrial hyperplasia or gynecological diseases that could be adversely affected by SERMs; (2) history of deep vein thrombosis or pulmonary embolism; (3) women who were unable to take bisphosphonate due to gastroesophageal reflux or gastrointestinal diseases; (4) women who were taking medication for osteoporosis; and (5) women who were taking medications that affected bone metabolism, such as steroids. Screening the inclusion and exclusion criteria were based on the patients' medical history and medical records.

\section{Randomization, treatment group assignment, and data collection}

For each study participant, the age, height, and weight were recorded prior to treatment, and the body mass index (BMI) was calculated from the height and weight measurements. Participants were randomly assigned to the alendronate or bazedoxifene group by block randomization method. Those in the alendronate group ingested a Fosamax Plus $D^{\circledR}$ tablet (91.37 mg sodium alendronate + $26.67 \mathrm{mg}$ cholecalciferol) once a week, and subjects in the bazedoxifene group ingested a Viviant tablet $(20 \mathrm{mg}$ bazedoxifene) every morning. All participants underwent a DXA scan (Lunar iDXA; GE Healthcare lunar, Madison, WI, USA) prior to and at the end of the 6-month treatment period. The DXA scans were performed by only one expert in the radiology department to reduce errors, and DXA reports were prepared by an experienced radiologist. The least significant change value of DXA scan in our hospital was $1.2 \%$. Markers for bone turnover were measured at the beginning of the study, at 3 months, and the end of the 6-month treatment period and included serum OC, vitamin D3, PTH, and CTX. Each blood sample was obtained at approximately $10 \mathrm{AM}$ after overnight fasting to exclude diurnal variation of the markers. Rates for medication adherence was analyzed via unannounced pill counts, and the survey was performed at the outpatient clinic. All participants were instructed to report any adverse side effects during the treatment period.

\section{Statistical analysis}

Descriptive statistics that included means and standard deviations (SDs) were conducted for all datasets. The Kolmogorov-Smirnov test was used for the determination of data normality. Continuous variables were compared be- 


\section{JBM}

tween the alendronate and bazedoxifene groups using a Mann-Whitney test, and comparisons of variables between time points within each group were performed using a Wilcoxon sign rank or Friedman test according to the number of comparisons. All statistical analyses were performed using SPSS version 20.0 (IBM Corp., Armonk, NY, USA), and statistical significance was set at $P$ less than 0.05 .

\section{RESULTS}

This study initially included 27 women with osteoporosis ( $T$ score $\leq-2.5$ on DXA), with 14 and 13 women assigned to the alendronate and bazedoxifene groups, respectively. There were 4 drop-outs in the alendronate group and 2 in the bazedoxifene group during the trial. Therefore, 21 women completed the study (Fig. 1). There were no significant differences between the 2 groups in terms of age, BMI, markers of bone turnover, and BMD prior to treatment. The drug adherence rate for alendronate group was 93.3\% (SD, $11.0 \%$ ) and the rate for bazedoxifene group was $88.7 \%$ (SD, $5.7 \%)$. There was no significant difference between the 2 groups (Table 1). No fracture occurred during follow-up.

\section{Changes in the markers of bone turnover during treatment}

The participants in the alendronate group showed significant decreases in the serum levels of $O C, 27.8$ to 12.7
$(P<0.001)$ and $\mathrm{CTX}, 0.43$ to $0.20(P=0.002)$ during the 6 months of treatment, while the bazedoxifene group showed a significant decrease only in serum OC levels, 22.5 to 16.1 $(P=0.012)$ (Table 2). At the 3-month timepoint, the CTX levels were less in the alendronate group than the bazedoxifene group. At the 6-month treatment timepoint, the

Table 1. Demographic data and measurements for participants prior to treatment with alendronate or bazedoxifene

\begin{tabular}{lccc}
\hline & $\begin{array}{c}\text { Alendronate } \\
\text { group (N=10) }\end{array}$ & $\begin{array}{c}\text { Bazedoxifene } \\
\text { group (N=11) }\end{array}$ & P-value \\
\hline Age (yr) & $64.2 \pm 8.0$ & $61.8 \pm 5.6$ & 0.435 \\
Height (cm) & $154.6 \pm 5.3$ & $153.4 \pm 4.5$ & 0.571 \\
Weight (kg) & $55.7 \pm 6.9$ & $61.5 \pm 11.1$ & 0.167 \\
BMI (kg/m²) & $23.3 \pm 2.8$ & $26.2 \pm 4.6$ & 0.107 \\
Drug adherence rate (\%) & $93.3 \pm 11.0$ & $88.7 \pm 5.7$ & 0.202 \\
BTM & & & \\
Osteocalcin (ng/mL) & $27.8 \pm 9.0$ & $22.5 \pm 8.0$ & 0.223 \\
C-telopeptide (ng/mL) & $0.43 \pm 0.22$ & $0.32 \pm 0.16$ & 0.314 \\
25(OH)D3 (ng/mL) & $45.5 \pm 10.5$ & $48.3 \pm 8.7$ & 0.605 \\
PTH (pg/mL) & $45.5 \pm 12.0$ & $38.4 \pm 5.5$ & 0.173 \\
BMD (g/cm²) & & & \\
L1-4 & $0.836 \pm 0.056$ & $0.884 \pm 0.086$ & 0.153 \\
Femur neck & $0.731 \pm 0.074$ & $0.751 \pm 0.058$ & 0.223 \\
Femur trochanter & $0.597 \pm 0.075$ & $0.637 \pm 0.073$ & 0.282 \\
Total femur & $0.780 \pm 0.069$ & $0.816 \pm 0.071$ & 0.282 \\
\hline
\end{tabular}

The data is presented as mean \pm standard deviation.

$\mathrm{BMI}$, body mass index; BTM, bone turnover marker; 25(OH)D3, 25-hydroxyvitamin D3; PTH, parathyroid hormone; BMD, bone mineral density.

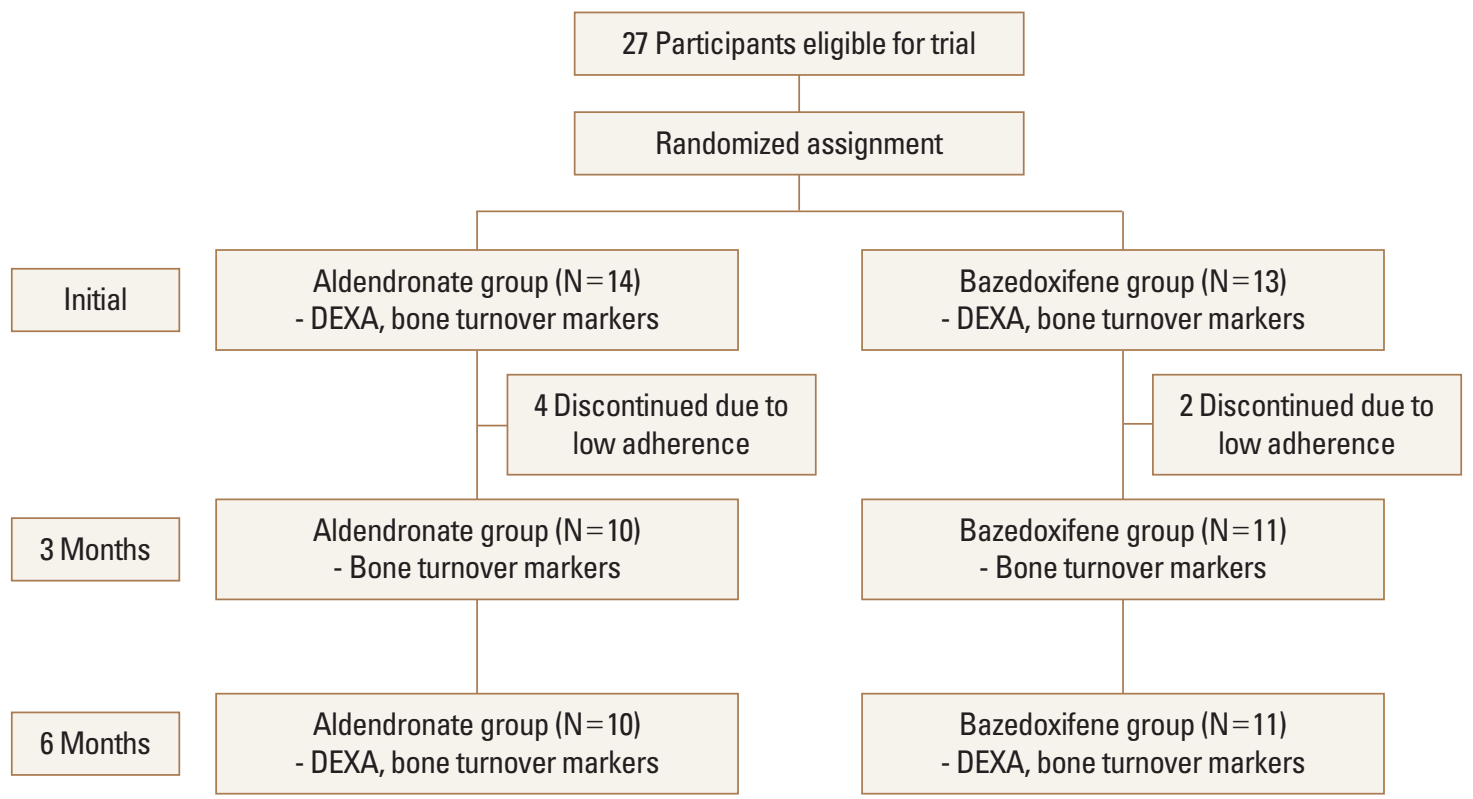

Fig. 1. Flow chart of participant recruitment for the randomized controlled trial to compare alendronate and bazedoxifene treatments. 
Table 2. Changes in markers of bone turnover during treatment

\begin{tabular}{|c|c|c|c|c|}
\hline & Baseline & 3 months & 6 months & $P$-value ${ }^{\text {a) }}$ \\
\hline \multicolumn{5}{|c|}{ Osteocalcin (ng/mL) } \\
\hline Alendronate & $27.8 \pm 9.0(100.0 \%)$ & $17.0 \pm 4.0(61.2 \%)$ & $12.7 \pm 4.2(45.7 \%)$ & $<0.001$ \\
\hline Bazedoxifene & $22.5 \pm 8.0(100.0 \%)$ & $18.3 \pm 6.6(81.3 \%)$ & $16.1 \pm 3.7(71.6 \%)$ & 0.012 \\
\hline$P$-value ${ }^{\mathrm{b}}$ & 0.223 & 0.705 & 0.020 & \\
\hline \multicolumn{5}{|c|}{ C-telopeptide (ng/mL) } \\
\hline Alendronate & $0.43 \pm 0.22(100.0 \%)$ & $0.15 \pm 0.11(34.9 \%)$ & $0.20 \pm 0.23(46.5 \%)$ & 0.002 \\
\hline Bazedoxifene & $0.32 \pm 0.16(100.0 \%)$ & $0.27 \pm 0.14(84.4 \%)$ & $0.34 \pm 0.15(106.3 \%)$ & 0.695 \\
\hline$P$-value ${ }^{b)}$ & 0.314 & 0.006 & 0.020 & \\
\hline \multicolumn{5}{|c|}{ 25(OH)D3 (ng/mL) } \\
\hline Alendronate & $45.5 \pm 10.5(100.0 \%)$ & $56.5 \pm 10.0(124.2 \%)$ & $51.4 \pm 14.6(123.0 \%)$ & 0.301 \\
\hline Bazedoxifene & $48.3 \pm 8.7(100.0 \%)$ & $52.8 \pm 11.6(109.3 \%)$ & $42.6 \pm 13.6(88.2 \%)$ & 0.148 \\
\hline$P$-value ${ }^{\text {b) }}$ & 0.605 & 0.654 & 0.197 & \\
\hline \multicolumn{5}{|l|}{ PTH (pg/mL) } \\
\hline Alendronate & $45.5 \pm 12.0(100.0 \%)$ & $43.0 \pm 14.8(94.5 \%)$ & $39.2 \pm 13.7(86.2 \%)$ & 0.301 \\
\hline Bazedoxifene & $38.4 \pm 5.5(100.0 \%)$ & $42.2 \pm 7.8(109.9 \%)$ & $43.3 \pm 15.2(112.8 \%)$ & 0.184 \\
\hline$P$-value ${ }^{\text {bl }}$ & 0.173 & 0.863 & 0.468 & \\
\hline
\end{tabular}

The data is presented as mean \pm standard deviation.

a) $P$-value were calculated using Mann-Whitney test. ${ }^{\text {b) }} P$-value were calculated using Friedman test.

25(OH)D3, 25-hydroxy-vitamin D3; PTH, parathyroid hormone.

Table 3. Changes in bone mineral density during treatment

\begin{tabular}{|c|c|c|c|}
\hline & Baseline & 6 months & $P$-value \\
\hline \multicolumn{4}{|l|}{ L1-4 BMD $\left(\mathrm{g} / \mathrm{cm}^{2}\right)$} \\
\hline Alendronate & $0.836 \pm 0.056(100.0 \%)$ & $0.871 \pm 0.071(104.2 \%)$ & 0.003 \\
\hline Bazedoxifene & $0.884 \pm 0.086(100.0 \%)$ & $0.891 \pm 0.083(100.8 \%)$ & 0.504 \\
\hline$P$-value ${ }^{\text {b) }}$ & 0.153 & 0.564 & \\
\hline \multicolumn{4}{|c|}{ Femur neck BMD $\left(\mathrm{g} / \mathrm{cm}^{2}\right)$} \\
\hline Alendronate & $0.731 \pm 0.074(100.0 \%)$ & $0.736 \pm 0.070(100.7 \%)$ & 0.610 \\
\hline Bazedoxifene & $0.751 \pm 0.058(100.0 \%)$ & $0.744 \pm 0.065(99.1 \%)$ & 0.167 \\
\hline$P$-value ${ }^{\text {b) }}$ & 0.223 & 0.739 & \\
\hline \multicolumn{4}{|c|}{ Femur trochanter BMD $\left(\mathrm{g} / \mathrm{cm}^{2}\right)$} \\
\hline Alendronate & $0.597 \pm 0.075(100.0 \%)$ & $0.620 \pm 0.078(107.1 \%)$ & 0.007 \\
\hline Bazedoxifene & $0.637 \pm 0.073(100.0 \%)$ & $0.631 \pm 0.075(99.1 \%)$ & 0.241 \\
\hline$P$-value ${ }^{\text {b) }}$ & 0.282 & 0.684 & \\
\hline \multicolumn{4}{|c|}{ Total femur BMD $\left(\mathrm{g} / \mathrm{cm}^{2}\right)$} \\
\hline Alendronate & $0.780 \pm 0.069(100.0 \%)$ & $0.792 \pm 0.067(101.5 \%)$ & 0.028 \\
\hline Bazedoxifene & $0.816 \pm 0.071(100.0 \%)$ & $0.811 \pm 0.075(99.4 \%)$ & 0.314 \\
\hline$P$-value ${ }^{\text {b) }}$ & 0.282 & 0.436 & \\
\hline
\end{tabular}

The data is presented as mean \pm standard deviation.

${ }^{a} P$-value were calculated using Mann-Whitney test. ${ }^{\text {b) }} P$-value were calculated using Wilcoxon sign rank test.

$\mathrm{BMD}$, bone mineral density.

serum levels of $\mathrm{OC}$ and $\mathrm{CTX}$ were less in the alendronate group than in the bazedoxifene group. There were no changes in serum levels of PTH and vitamin D3 observed during the treatments.

\section{Changes in BMD during treatment}

The majority of bone sites examined by DXA showed significant increases in BMD after 6 months of alendronate treatment from 0.836 to 0.871 in L1-4 average, while the bazedoxifene group did not show any significant increase 
in BMD from 0.884 to 0.891 (Table 3). However, there was no significant increase in BMD in the bazedoxifene group. There were no significant differences observed in BMDs between the 2 groups at the pretreatment and 6-month timepoints for the body sites examined.

\section{DISCUSSION}

The aim of this study was to evaluate the short-term changes in osteogenic and osteoclastic biomarkers and BMD after administration of 2 different types of medications, alendronate and bazedoxifene, for osteoporosis. Bazedoxifene showed slower suppression of bone metabolism compared to alendronate. After 6 months of treatment, the alendronate treatment group showed a significant decrease in bone resorption and formation markers. The bazedoxifene treatment group showed a significant decrease only in a bone formation marker. The alendronate group showed significant increases in BMD, as observed by DXA scan, at multiple bone sites after 6 months of treatment, while the bazedoxifene group did not. However, the BMDs between the 2 groups were not significantly different at pre- or post-treatment timepoints. This was the first clinical study to compare the efficacy of alendronate and bazedoxifene on osteoporosis by measuring BMD and bone turnover markers during a short-term follow-up.

In the alendronate group, levels of serum OC decreased by 6 months of treatment, while CTX levels decreased by a significant amount in the first 3 months and then by a smaller amount in the next 3 months, forming almost a nadir. This finding is consistent with a previous study that reported a decrease in bone resorption markers within 6 weeks to 3 months after the initiation of anti-resorptive therapy, while the nadir in bone formation markers was delayed until 6 months.[7] The rapid reduction in serum levels of CTX compared with OC after alendronate treatment demonstrated the rapid suppression of bone resorption as the primary action of this drug. Reflecting this rapid change of bone turnover markers, BMD significantly increased only after 6 months of treatment in the alendronate group. In addition to a previous study that reported the effect of alendronate on BMD at a median follow-up of 4 years,[8] our study showed that this drug significantly increased BMD during short-term administration.

In the bazedoxifene treatment group, the serum levels of OC decreased after 6 months of treatment, while CTX levels did not show a significant change. Furthermore, 6 months of bazedoxifene treatment did not induce significant changes in BMD. Our study period may have been too short to evaluate the clinical effects of bazedoxifene on osteoporosis considering that a previous study reported that bazedoxifene significantly increased BMD and reduced bone turnover after 5 years of treatment to those observed in the placebo group.[9]

Bjarnason et al. [10] reported that changes in bone turnover were related to fracture risk during 3 years of raloxifene therapy; however, changes in BMD were not associated with fracture risk. Although bone quantity, as measured by DXA, is an important factor contributing to bone strength, bone quality is another component that is potentially important for bone strength and fracture risk. Determination of bone quality can be performed by examining trabecular bone score and micro computed tomography, but they are not widely used. The disparity between bone turnover rate, fracture risk, and bone quantity leads us to focus on the potential role of currently unknown bone quality measurements.

Bone is a complex connective tissue whose homeostasis is maintained by an equilibrium between bone formation and resorption. Rapid and strong suppression of bone turnover by bisphosphonates is considered beneficial in preserving or increasing BMD.[11] However, excessive suppression of bone turnover may cause accumulation of bone with inappropriate quality, which may result in atypical fractures in spite of increased BMD.[12] Bazedoxifene treatment has not been shown to cause atypical fractures. Furthermore, previous network meta-analysis showed bazedoxifene showed higher efficiency in preventing vertebral fractures than bisphosphonates in groups of individuals at high risk for osteoporotic fractures who had Fracture Risk Assessment Tool scores > 20\%.[13] Therefore, bazedoxifene may be more beneficial for preserving bone quality than alendronate. In this context, the different bone turnover patterns between alendronate and bazedoxifene shown in our study prompt the clinical question of what the optimal bone turnover rate should be for better bone quality. Further study is required to determine whether bazedoxifene's slower suppression of bone turnover may be a factor in determining better bone quality.

Drug adherence in the alendronate group and the bazedoxifene group were not significantly different. There were 
4 dropouts in the alendronate group and 2 dropouts in the bazedoxifene group. In the alendronate group, 2 participants dropped out owing to possible side effects and the other 2 due to concurrent treatment of other diseases. In the bazedoxifene group, the participants dropped out due to concurrent treatment of other diseases. They were both oral tablets, but the frequency of the medication was different, with alendronate being administered daily and bazedoxifene once a week. Despite the small number of participants in the study, the frequency of the tablets did not affect the drug adherence rate.

Limitations of the study need to be considered while interpreting the study results. First, this study had a small sample size, and the study results need to be generalized with caution. Second, bone turnover markers are subject to circadian variability. Although the blood samples were obtained at approximately $10 \mathrm{AM}$ to reduce the diurnal variation, there may have been an unknown bias measuring these markers. Third, the study was conducted over a short period. Six months are not sufficient to monitor change in BMD and long-term effect of osteoporotic medication. Long-term study is needed in the future.

In conclusion, during 6 months of treatment, alendronate showed a more rapid suppression of bone turnover than bazedoxifene, and this likely caused significant increases in BMDs in the alendronate group. Considering the long-term clinical effects of both drugs on BMD and fracture rate, the relationship between the bone turnover rate and bone quality needs to be investigated in future studies.

\section{DECLARATIONS}

\section{Funding}

This research was supported by the Ministry of Trade, Industry and Energy of Korea (Grant no. 10045220).

\section{Ethics approval and consent to participate}

This study complies with the Declaration of Helsinki and was performed according to ethics committee approval. All patients provided written informed consent prior to enrollment.

\section{Conflict of interest}

No potential conflict of interest relevant to this article was reported.
ORCID

Hee Soo Han https://orcid.org/0000-0002-8865-1718

Moon Seok Park https://orcid.org/0000-0002-2856-7522

Ki Hyuk Sung https://orcid.org/0000-0002-5007-2403

Kyoung Min Lee https://orcid.org/0000-0002-2372-7339

\section{REFERENCES}

1. Migliaccio S, Brama M, Spera $G$. The differential effects of bisphosphonates, SERMS (selective estrogen receptor modulators), and parathyroid hormone on bone remodeling in osteoporosis. Clin Interv Aging 2007;2:55-64. https:// doi.org/10.2147/ciia.2007.2.1.55.

2. Garnero P. Bone markers in osteoporosis. Curr Osteoporos Rep 2009;7:84-90. https://doi.org/10.1007/s11914-0090014-3.

3. International Atomic Energy Agency. Dual energy X ray absorptiometry for bone mineral density and body composition assessment. IAEA Human Health Series No. 5. Vienna, AT: International Atomic Energy Agency; 2011.

4. Kuchuk NO, van Schoor NM, Pluijm SM, et al. Vitamin D status, parathyroid function, bone turnover, and BMD in postmenopausal women with osteoporosis: global perspective. J Bone Miner Res 2009;24:693-701. https://doi. org/10.1359/jbmr.081209.

5. Jordan N, Barry M, Murphy E. Comparative effects of antiresorptive agents on bone mineral density and bone turnover in postmenopausal women. Clin Interv Aging 2006; 1:377-87. https://doi.org/10.2147/ciia.2006.1.4.377.

6. Rejnmark L, Mosekilde L. New and emerging antiresorptive treatments in osteoporosis. Curr Drug Saf 2011;6:7588. https://doi.org/10.2174/157488611795684686.

7. Ebeling PR, Akesson K. Role of biochemical markers in the management of osteoporosis. Best Pract Res Clin Rheumatol 2001;15:385-400. https://doi.org/10.1053/berh.2001. 0156.

8. Donaldson MG, Palermo L, Ensrud KE, et al. Effect of alendronate for reducing fracture by FRAX score and femoral neck bone mineral density: the Fracture Intervention Trial. J Bone Miner Res 2012;27:1804-10. https://doi.org/10.1002/ jbmr.1625.

9. Silverman SL, Christiansen C, Genant HK, et al. Efficacy of bazedoxifene in reducing new vertebral fracture risk in postmenopausal women with osteoporosis: results from a 3-year, randomized, placebo-, and active-controlled 
clinical trial. J Bone Miner Res 2008;23:1923-34. https:// doi.org/10.1359/jbmr.080710.

10. Bjarnason NH, Sarkar S, Duong T, et al. Six and twelve month changes in bone turnover are related to reduction in vertebral fracture risk during 3 years of raloxifene treatment in postmenopausal osteoporosis. Osteoporos Int 2001; 12:922-30. https://doi.org/10.1007/s001980170020.

11. Lee SY, Kim KM, Kong SH, et al. The effects of low-dose bisphosphonate treatment on bone mineral density and bone turnover markers in elderly patients with osteoporosis. Ann Geriatr Med Res 2016;20:131-6. https://doi.org/ 10.4235/agmr.2016.20.3.131.

12. Saita Y, Ishijima M, Kaneko K. Atypical femoral fractures and bisphosphonate use: current evidence and clinical implications. Ther Adv Chronic Dis 2015;6:185-93. https:// doi.org/10.1177/2040622315584114.

13. Ellis AG, Reginster JY, Luo X, et al. Indirect comparison of bazedoxifene vs oral bisphosphonates for the prevention of vertebral fractures in postmenopausal osteoporotic women. Curr Med Res Opin 2014;30:1617-26. https://doi. org/10.1185/03007995.2014.908279. 
